Artículo

\title{
Evaluación de la intensidad del tráfico de tractores e implementos en un suelo agrícola
}

\author{
Enrique García Rodríguez ${ }^{1}$ \\ Francisco Gutiérrez-Rodríguez ${ }^{2 \S}$ \\ José Francisco Ramírez Dávila ${ }^{2}$ \\ Andrés González-Huerta ${ }^{2}$ \\ Delfina de Jesús Pérez López ${ }^{2}$ \\ Rodolfo Serrato Cuevas ${ }^{2}$ \\ ${ }^{1}$ Ciencias Agropecuarias y Recursos Naturales-Universidad Autónoma del Estado de México. ${ }^{2}$ Centro de \\ Investigación y Estudios Avanzados en Fitomejoramiento-Facultad de Ciencias Agrícolas-Universidad \\ Autónoma del Estado de México. Centro Universitario 'El Cerrillo', El Cerrillo Piedras Blancas, Toluca, \\ Estado de México. CP. 50200. Tel. 722 296 5529. (egarciar0105@hotmail.com; framirezd@uaemex.mx; \\ agonzalezh@uaemex.mx; seccum@yahoo.com.mx; djperezl@aemex.mx).
}

Autor para correspondencia: fgrfca@hotmail.com.

\section{Resumen}

Diversos autores, establecen que la compactación del suelo se considera como el mayor problema ambiental originado por la agricultura convencional, es el tipo de degradación más difícil de localizar y entender, porque no muestra marcas evidentes en la superficie del suelo. Para conocer la intensidad de las pasadas y su efecto sobre la compactación o índice de cono del conjunto máquina tractor durante el ciclo agrícola del maíz, con las tecnologías de labranza convencional y mínima, se equipó a cada tractor con un receptor DGPS de señales al entrar al campo de cultivo, para monitorear el tránsito en todas las labores, y los datos se registraron con una frecuencia de 2 s. Se midió el ancho de los neumáticos observando la huella reflejada en el suelo con una presión de aire de $140 \mathrm{kPa}$. En labranza tradicional con arado de discos se cubrió $91.15 \%$ y en total fue $384.83 \%$ de la superficie. En la tecnología de labranza mínima con el multiarado se cubrió 36.95\% y en total fue de $170.08 \%$. Siendo ostensiblemente menor en esta tecnología que en la labranza tradicional. En lo referente a Índice de cono (Ic) en la labranza con arado de discos después de la siembra el Ic supero los $3 \mathrm{MPa}$ después de la profundidad de $12.5 \mathrm{~cm}$ mientras que con multiarado en $80 \%$ de los datos están por debajo de los mismos $3 \mathrm{MPa}$, coincidiendo con el Ic después de la cosecha en ambos casos.

Palabras claves: arado de disco, compactación del suelo, DGPS, multiarado, rastra, sembradora, sistema de rodaje.

Recibido: marzo de 2020

Aceptado: junio de 2020 


\section{Introducción}

La ingeniería de suelos define a la compactación como el proceso mediante el cual las partículas del suelo son reacomodadas para disminuir el espacio poroso y colocarlas más cerca unas de otras, ocasionando un incremento de la densidad aparente. En un sentido agronómico, la compactación es el resultado de la aplicación al suelo de cualquier fuerza, por ejemplo, la ejercida por la labranza y el tránsito rodado de maquinaria agrícola, que incrementa la densidad aparente del suelo y concurrentemente, disminuye la porosidad de este (Soil Science Society of America, 2013).

En suelos densos o compactados se impide el crecimiento de las raíces y de este modo se limita el consumo de agua a la planta, lo que afecta su rendimiento al paso reiterado de las máquinas agrícolas. Según plantea Martiren et al. (2016), solo en Europa 33000000 ha de suelo arable se encuentra altamente degradado por el reiterado paso del sistema de rodaje de los tractores agrícolas. La FAO (2015) establece las 10 principales amenazas a las funciones del suelo: erosión, pérdida de carbono orgánico, desequilibrio de nutrientes, acidificación del suelo, contaminación, anegamiento, compactación del suelo, sellado, salinización y pérdida de la biodiversidad del suelo.

Por otro lado, Cerisola et al. (2015) establece que el gran desafío es el diagnóstico de la calidad de vida de los suelos para desarrollar un sistema productivo, basado en una agricultura sostenible y plantea que las mejores condiciones para el tráfico son innegables, siempre que sean controlados, pues el tráfico trae como consecuencia modificaciones físicas que conducen a la compactación. Draghi et al. (2015) plantea que es notorio que el tráfico sobre suelo labrado provoca $83 \%$ del hundimiento final en la primera pasada. El tráfico posterior y hasta las diez pasadas provoca sólo $17 \%$ del hundimiento restante.

En definitiva, la no labranza evidencia un suelo con mayor capacidad portante, pero sensible a la intensidad del tráfico, aún más que el suelo labrado. La compactación del suelo también afecta a la mineralización del carbono (C) y nitrógeno $(\mathrm{N})$ de la materia orgánica del suelo y del rastrojo (Neve y Hofman, 2000), así como a la concentración del dióxido de carbono $\left(\mathrm{CO}_{2}\right)$ en el suelo (Conlin y Driessche, 2000).

En la actualidad la compactación es considerada un problema medioambiental muy grave causado por prácticas tecnológicas en la agricultura convencional, es muy difícil localizarlo y racionalizarla, porque las marcas del sistema de rodaje de los tractores y las máquinas agrícolas en la superficie del suelo no son evidentes (McGarry, 2001). A diferencia de la salinización y la erosión que proporcionan una fuerte evidencia superficial de la presencia de degradación de la tierra, la degradación de la estructura del suelo requiere de monitoreo físico y examinación antes de ser descubierta, y su extensión, naturaleza y causa, resueltas (Hamza y Anderson, 2005).

La compactación del suelo es causada por la alta intensidad del tráfico y la presión de los neumáticos sobre el suelo del tractor y se combina en la cosecha, especialmente cuando estas operaciones se llevan a cabo en suelo húmedo o con neumáticos de alta presión sobre el suelo. Según Kirkegaar (1990) el efecto de la dureza del suelo en la raíz daña el crecimiento de esta en la mayoría de las especies y reduce el crecimiento $50 \%$, cuando el Ic se encuentra de 0.7 a $1.5 \mathrm{MPa}$ y se limita completamente a valores mayores de $4 \mathrm{MPa}$. 
En ensayos realizados se ha utilizado un receptor GPS (sistema de posicionamiento global) como equipo simple el cual se coloca en un tractor y puede detectar las áreas en el campo donde aparecen las huellas del paso del sistema de rodaje de las máquinas agrícolas durante el trabajo de esta. Richards (2000) utilizó el sistema de posicionamiento global basado en tractores para mapear todos los movimientos del vehículo dentro de un campo durante un ciclo agrícola.

Es necesario destacar la naturaleza oculta de la degradación estructural del suelo (DES), la cual conlleva problemas específicos como, un pobre crecimiento del cultivo o infiltración del agua, que pudieran ser atribuidos a otras causas. Adicionalmente, a la DES puede señalársele como culpable por un pobre desempeño del cultivo cuando de hecho no está presente (Hamza y Anderson, 2005).

En este sentido, debido a que la compactación del subsuelo es muy persistente y las posibilidades naturales o artificiales de su aflojamiento han resultado decepcionantes, la Unión Europea (UE) la ha reconocido como una forma severa de degradación del suelo (Akker y Canarache, 2001). Wild (1992), plantea que la compactación y consolidación del suelo, acompañada por la pérdida de los poros más grandes, es el resultado de la deformación y rotura bajo carga de los agregados y poros del suelo, que conduce a una pérdida de permeabilidad para el agua y las raíces.

Con altos contenidos de agua, el suelo usualmente se deforma fácilmente, puede darse poca compactación a menos que haya tiempo y oportunidad para que el agua escape. Con suelos húmedos, bajo las cargas transitorias producidas por el tráfico y algunos aperos de labranza, puede haber encharcamiento, deformación, pérdidas de agregados y quizás algo de dispersión, pero poca pérdida de volumen de suelo. Sin embargo, al secarse, el suelo se vuelve intrínsecamente más fuerte y la susceptibilidad a la compactación puede aumentar pues los poros más amplios, que se vacían primero, son relativamente débiles.

Así, con el aumento del secado, el aumento de la resistencia del suelo se hace predominante. Por lo que, en suelos contraíbles/expandibles, la densidad aparente debería ser determinada a contenidos de humedad estandarizados, para prevenir problemas originados por las variaciones en el contenido de agua (Håkansson y Lipiec, 2000). Por otro lado, la dureza del suelo se usa como medida de la compactación porque refleja la resistencia del suelo a la penetración de raíces (Hamza y Anderson, 2005).

En cuanto a la tasa de infiltración de agua en el suelo, ésta también puede ser usada para monitorear el estatus de la compactación, especialmente de la capa superficial, ya que el agua infiltra suelos no compactados que tienen partículas de suelo bien agregadas mucho más rápido que suelos pesados, con menor estructura (Hamza y Anderson, 2005). Bouwman y Arts (2000) sostienen que un ligero grado de compactación superficial puede ser benéfico para algunos tipos de suelo, indicando de manera que existe un nivel óptimo de compactación para el crecimiento del cultivo.

El concepto de nivel óptimo de compactación es importante, especialmente en sistemas de tránsito controlado, donde se evita cualquier fuente externa de compactación porque ésta podría causar un nivel de compactación menor al óptimo y decrementos en el rendimiento. Con la finalidad de evaluar el tránsito de los tractores por el campo de cultivo se eligieron dos métodos de labranza, con el objetivo de conocer la cantidad de veces que en la preparación del suelo los tractores hacen sus respectivas pasadas y en que magnitud cubren la superficie de la parcela objeto de la investigación. 


\section{Materiales y métodos}

El presente estudio se realizó de abril 2018 a marzo 2019 en la Facultad de Ciencias Agrícolas de la Universidad Autónoma del Estado de México, ubicada a los $19^{\circ} 23^{\prime}$ '30', latitud norte y $99^{\circ} 41^{\prime}$ 30" a una altitud de $2640.5 \mathrm{~m}$. En el Campus Universitario la superficie total disponible es de 110 ha. La clasificación del suelo, de acuerdo USDA (2013), se encuentra en el orden vertisol y dentro del subgrupo Entic pelluderts, con un contenido de arcilla superior a $30 \%$ hasta los $50 \mathrm{~cm}$ de profundidad.

El terreno utilizado para el ensayo tenía cinco años sin utilización agrícola y los parámetros escogidos, fueron el mapeo de las pasadas del sistema de rodaje de los tractores registradas por medio de receptor de señales de DGPS. Las tecnologías de preparación del suelo utilizadas fueron, la labranza convencional del suelo basada en el arado de discos, que tradicionalmente consta de un pase de rastra, un pase del arado de discos, una o dos pases de rastra, siembra y una o dos pases de escara o cultivo, en una superficie de 16.41ha así como labranza mínima o reducida, que consta de un pase de rastra, un pase del multiarado, un pase de rastra, la siembra y una escarda, utilizando el multiarado con una superficie de $17.51 \mathrm{ha}$. La profundidad promedio de la rastra es de $15 \mathrm{~cm}$ de profundidad, el arado de discos con una media de $20 \mathrm{~cm}$ de profundidad, la siembra a $12 \mathrm{~cm}$ de profundidad, la cultivadora a $20 \mathrm{~cm}$ de profundidad y el multiarado de $35 \mathrm{~cm}$ de profundidad en promedio.

Estas labores fueron simultáneas en ambas parcelas, en la rastra rompe surcos, rastra rompe terrones, posterior al arado de disco o el multiarado, la siembra y las escardas. En una parcela se utilizó el arado de discos y en la otra el multiarado simultáneamente. Las características de los tractores y los implementos utilizados para las labores primarias y complementarias (siembra y cultivo) en ambas tecnologías, se muestran en el Cuadro 1, donde la determinación de la masa se realizó en una báscula pública donde $50 \%$ de la parte delantera se encuentra el centro de gravedad del tractor y de esa misma manera la parte trasera, donde se obtuvo que aproximadamente $40 \%$ de su masa se encuentra en la parte delantera y $60 \%$ restante en la parte trasera, libres de contrapesos, como lo estipula el fabricante, para con posterioridad agregar el líquido y sólido a las llantas, también como estipula el fabricante para una buena operación en campo.

Cuadro 1. Características técnicas de los tractores empleados en el ensayo. Toluca, México, 20182019.

\begin{tabular}{lllll}
\hline $\begin{array}{l}\text { Modelo del } \\
\text { tractor }\end{array}$ & $\begin{array}{l}\text { Masa del } \\
\text { tractor } \\
(\mathrm{kg})\end{array}$ & $\begin{array}{l}\text { Medida de las } \\
\text { ruedas traseras y } \\
\text { delanteras } \\
\text { (pulgadas) }\end{array}$ & $\begin{array}{l}\text { Presión media específica } \\
\text { sobre el suelo rueda } \\
\text { trasera (presión } \\
\text { neumático140 kPa) }\end{array}$ & $\begin{array}{l}\text { Presión media específica } \\
\text { sobre el suelo rueda } \\
\text { delantera (presión } \\
\text { neumático140 kPa) }\end{array}$ \\
\hline $\begin{array}{l}\text { 6603 John Deere } \\
\text { (4X4) neumático } 5872\end{array}$ & $\begin{array}{l}18.4-34 \\
\text { radial }\end{array}$ & $14.9-24$ & $249 \mathrm{kPa}($ ambas ruedas & $177 \mathrm{kPa}$ (ambas ruedas \\
& & $498 \mathrm{kPa})$ & $354 \mathrm{kPa})$ \\
$\begin{array}{l}\text { 5715 John Deere } \\
\text { (4X4) neumático } 2790\end{array}$ & $15.5-38$ & & \\
radial & $7.50-16$ & $324 \mathrm{kPa})$ & $90.5 \mathrm{kPa}$ (ambas ruedas \\
\hline
\end{tabular}


Los tractores utilizados fueron instrumentados con un receptor de señales DGPS modelo Oregón 650 Garmin, el cual enviaba una señal cada $2 \mathrm{~s}$, almacenada en el dispositivo de la memoria de medición y con estos datos se realizó el mapeo del tráfico. El mapeo de suelos fue realizado y los datos contabilizados con el programa MapSource. La intensidad del tráfico del conjunto tractorimplemento fue monitoreada en las dos tecnologías de labranza del suelo mencionadas. Las tomas de muestra del suelo se realizaron en pre-siembra, en la siembra y en la cosecha, para determinar la densidad aparente, la humedad y la textura.

Todos los tractores utilizados tenían ruedas sencillas en los dos ejes. En los trabajos de preparación de suelos, en cada aplicación de cada tecnología, los tractores utilizados estaban dotados de lastre sólido y liquido en las ruedas motrices y este último hasta $75 \%$ de su capacidad con agua, aunque esto aumenta de 5 a $10 \%$ el consumo de combustible por unidad de suelo elaborada también disminuye el patinaje de las ruedas motrices y aumenta la compactación del suelo.

No obstante, se debe plantear que bajo estas condiciones de diseño se trabajan tradicionalmente los tractores en cada ciclo agrícola y bajo dichas condiciones se realizaron los experimentos. Durante el ensayo el Ic se determinó con el uso de un penetrómetro Scout 900 S313 (ASAE Standards S313.2, 1993). Para determinar la superficie cubierta por la maquinaria e implementos, se hace una simple operación aritmética: ancho de llantas (las 2) por largo de la parcela por número de pasadas del conjunto máquina-tractor.

Se hace una toma de muestras en pre-siembra, en cada uno de los 25 puntos predeterminados, donde se determina la densidad aparente (DA), índice de cono (Ic). Con posterioridad, se tomaron muestras de suelo a cada $10 \mathrm{~cm}$ de profundidad, esto es, de 0-10, 10-20 y 20-30 cm de profundidad en 25 puntos preestablecidos en cada parcela, en la siembra y en la cosecha.

\section{Resultados y discusión}

Se realizaron muestreos de suelo antes de las labores de preparación primaria del suelo, donde la densidad aparente (DA) arrojo un promedio de a $1.43 \mathrm{Mg} \mathrm{m}^{-3}$, el Índice de cono (Ic) no pudo ser medido pues en la capa de 0 a $10 \mathrm{~cm}$ de profundidad fue de más de $7 \mathrm{MPa}$, superando lo que podía medir el compactómetro y en las profundidades mayores de $10 \mathrm{~cm}$ fue de $6 \mathrm{MPa}$ como promedio. En el Cuadro 2 se muestran los diferentes trabajos realizados con el conjunto tractor-máquina en la tecnología de labranza tradicional, los cuales fueron de ocho pasadas con los diferentes implementos y con diferentes tractores en los trabajos mecanizados.

Cuadro 2. Frecuencia de tránsito dado en porcentaje, en la parcela con tecnología de labranza convencional. Toluca, México, 2018-2019.

\begin{tabular}{ccccc}
\hline $\begin{array}{c}\text { Tipos de } \\
\text { implementos }\end{array}$ & $\begin{array}{c}\text { Ancho del } \\
\text { implemento }(\mathrm{m})\end{array}$ & $\begin{array}{c}\text { Ancho de la rueda } \\
\text { trasera del tractor }(\mathrm{m})\end{array}$ & $\begin{array}{c}\text { Superficie } \\
\text { cubierta (ha) }\end{array}$ & $\begin{array}{c}\text { Frecuencia del } \\
\text { tráfico }(\%)\end{array}$ \\
\hline $\begin{array}{c}1^{\text {ra }} \text { rastra (tractor } \\
6603)\end{array}$ & 3 & $0.41($ ambas 0.82$)$ & 6.02 & 36.7 \\
$\begin{array}{c}\text { da } \\
\text { rastra (tractor } \\
6603)\end{array}$ & 3 & $0.41(0.82)^{*}$ & 6.17 & 37.6 \\
$3^{\text {ra }} \begin{array}{c}\text { rastra (tractor } \\
6603)\end{array}$ & 3 & $0.41(0.82)^{*}$ & 9.85 & 60 \\
\hline
\end{tabular}




\begin{tabular}{ccccc}
\hline $\begin{array}{c}\text { Tipos de } \\
\text { implementos }\end{array}$ & $\begin{array}{c}\text { Ancho del } \\
\text { implemento }(\mathrm{m})\end{array}$ & $\begin{array}{c}\text { Ancho de la rueda } \\
\text { trasera del tractor }(\mathrm{m})\end{array}$ & $\begin{array}{c}\text { Superficie } \\
\text { cubierta }(\mathrm{ha})\end{array}$ & $\begin{array}{c}\text { Frecuencia del } \\
\text { tráfico }(\%)\end{array}$ \\
\hline $\begin{array}{c}\text { Arado de disco } \\
\text { tractor 5715) } \\
4^{\text {ta }} \text { rastra (tractor } \\
6603)\end{array}$ & 1.02 & $0.47(0.94)^{*}$ & 14.96 & 91.1 \\
$\begin{array}{c}\text { Siembra (tractor } \\
6603)\end{array}$ & 3 & $0.41(0.82)^{*}$ & 11.73 & 71.5 \\
$1^{\text {ra }} \begin{array}{c}\text { escarda (tractor } \\
5715)\end{array}$ & 3.2 & $0.41(0.82)^{*}$ & 4.41 & 26.9 \\
$2^{\text {da }} \begin{array}{c}\text { escarda (tractor } \\
5715)\end{array}$ & 2.4 & $0.47(0.94)^{*}$ & 5.33 & 32.5 \\
Total & 2.4 & $0.47(0.94)^{*}$ & 4.66 & 28.4 \\
\hline
\end{tabular}

*= ancho de ambas ruedas traseras del tractor utilizado.

El ancho real de las ruedas motrices que se tomaron en cuenta fue de $0.86 \mathrm{~m}$ (ambas $1.72 \mathrm{~m}$ ), y después de contabilizar el número de pasadas por la parcela del tractor con el implemento, la superficie cubierta fue de 6.03 ha, equivalente a $36.7 \%$ de cubrimiento de la superficie de cultivo por el sistema de rodaje del tractor y de esta manera se pasó tres veces la rastra a una profundidad promedio de $15 \mathrm{~cm}$, lo cual conllevó a una superficie total de 22.04 ha, equivalente al $134.4 \%$ del total de la superficie removida.

En el Cuadro 2 se ponen las diferentes labores que se realizaron en orden cronológico durante el trabajo que se llevó a efecto en el campo durante la preparación primaria y complementaria del suelo, se pasaron por el campo tres veces la rastra lo cual se realizó en función de destruir los alomados producidos por el cultivo de las plantas de maíz en años anteriores y dejarlos lo más llano posible para que pudiera trabajar el arado de discos a una profundidad de $20 \mathrm{~cm}$, en las condiciones más óptimas posibles.

En la segunda pasada de rastra se cubrió $37.6 \%$ de la superficie y en la tercera pasada de rastra se cubrió $60 \%$ y en esta última a veces se volvía a pasar casi por la misma huella anterior dejada por el sistema de rodaje del tractor y no hubo traslapo, por lo que pudo ser contabilizada y calculada la superficie dejada por el sistema de rodaje del tractor y registrada por el DGPS y esta última llego a ser de 60\%. Asimismo, todas las líneas registradas por el DGPS en todos los casos fueron evaluadas de la misma manera para las dos tecnologías de preparación de suelos, sin excluir ninguna operación por básica que fuese.

Es importante señalar, que las tres primeras operaciones de rastra y que aparecen con un asterisco, no son parte tradicional de esta tecnología de preparación de suelos, pero es muy utilizada cuando los suelos Vertisoles en la zona del valle de Toluca se dejan de cultivar por espacio de uno o más ciclos agrícolas, ya que los mismos pueden alcanzar en la capa arable resistencia a la penetración que puede sobre pasar los $5 \mathrm{MPa}$, estas operaciones no son normales o recurrentes en esta tecnología, y que su aplicación depende del uso anterior del suelo. 
En la labor de aradura se puede observar que el arado de disco tiene un ancho de corte bastante pequeño $(1.02 \mathrm{~m})$, lo cual aumentó ostensiblemente el número de pasadas del conjunto máquinatractor sobre la superficie de labranza y al contabilizarlas en el mapa, el transito del sistema de rodaje del tractor sobre el suelo fue de $91.1 \%$, lo que contribuyó a incrementar la compactación, lo cual es coincidente con algunos investigadores (Tullberg et al., 2007; Tullberg, 2010).

En la Figura 1 (izquierda) se observa la cantidad de pasadas que se realizaron con el arado de discos y en la cual el conjunto tractor -implemento se desplaza de forma paralela en cada una de las pasadas y no existen puntos de concordancia, pero existe una gran densidad de estos, pues cada $1.02 \mathrm{~m}$ tiene que pasarse el implemento para poder remover toda la superficie de suelo en esta tecnología. En el Cuadro 2, se puede apreciar que se utilizó la rastra posterior a la labor de aradura, en función de buscar un buen mullido o destrucción de todos los terrones del suelo.

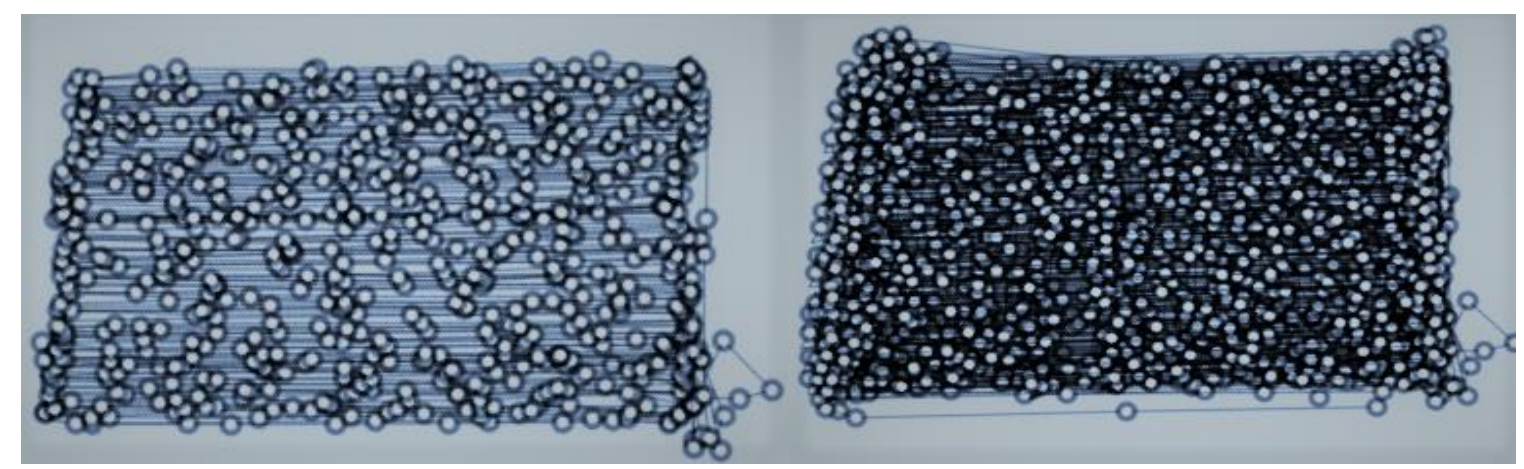

Figura 1. Tránsito de los tractores y la maquinaria agrícola para la tecnología convencional de labranza de suelo en 1.64 ha. Izquierda= recorrido del tractor con arado de discos; derecha= trayectorias de movimiento en el campo durante el ciclo completo de cultivo del maíz. Toluca, México, 2018-2019.

Lo cual ocasiono que el sistema de rodaje del tractor transitara de nuevo por la superficie en elaboración $71.5 \%$, lo cual es altamente destructivo para la estructura físico-mecánica del suelo, así como un aumento en DA y una disminución en la productividad del suelo, lo cual es coincidente con lo planteado por Laureda et al. (2016). En la Figura 1 (derecha) se puede observar la densidad de las pasadas en toda la superficie elaborada en esta tecnología, donde no existe un punto donde no hayan pasado las ruedas del tractor y donde se tienen alrededor de 500 puntos de concordancia y en número de dos repeticiones por lo menos y hasta cinco en el mismo punto como máximo.

En esta figura se sobre pusieron los ocho mapas que se obtuvieron de las diferentes operaciones tecnológicas de preparación del suelo. En las últimas cinco operaciones mecanizadas (Cuadro 2) se transitó por encima de la superficie en la preparación del suelo un total de $250.3 \%$, lo cual es un valor apreciable, máxime si se tiene en cuenta que es un suelo Vertisol, que es de los más compactos y con mucha susceptibilidad al aumento de la densidad aparente al paso del sistema de rodaje de los tractores en las diferentes labores mecanizadas.

Es importante, observar los resultados del cálculo de la superficie cubierta por el sistema de rodaje mostrado en el Cuadro 2 y de acuerdo a lo planteado por Hamza y Anderson (2005), la labranza convencional fue evaluada por estos investigadores como la más dañina de las tecnologías de las preparaciones de suelo, y en el caso de esta investigación, la concentración observada en la Figura 1 (derecha) las pasadas reiteradas del sistema de rodaje de los tractores es una de las desventajas más importantes, fundamentalmente en suelos Vertisoles. 
En la Figura 2a se presenta el comportamiento del Ic después de la siembra del maíz con la preparación del suelo con arado de discos. Es necesario, plantear que antes de aplicar el arado de discos se aplicó por tres veces la rastra de discos para destruir los camellones que quedaron cinco años atrás y hasta los $10 \mathrm{~cm}$ de profundidad el Ic fue de $1.22 \mathrm{MPa}$ y densidad aparente (DA) de $1.21 \mathrm{Mg} \mathrm{m}^{-3}$; a partir de los $12.5 \mathrm{~cm}$ fue de $2.16 \mathrm{MPa}$, donde el sistema radical de las plantas no alcanza un buen crecimiento y llegando alcanzar a los $30 \mathrm{~cm}$ un Ic de $5.84 \mathrm{MPa}$ y DA de $1.21 \mathrm{Mg}$ $\mathrm{m}^{-3}$, lo cual es coincidente con lo planteado por donde también indica que en suelos extremadamente densos el crecimiento de las raíces se limita, así como también el consumo de agua a la planta y por ende afecta el rendimiento.

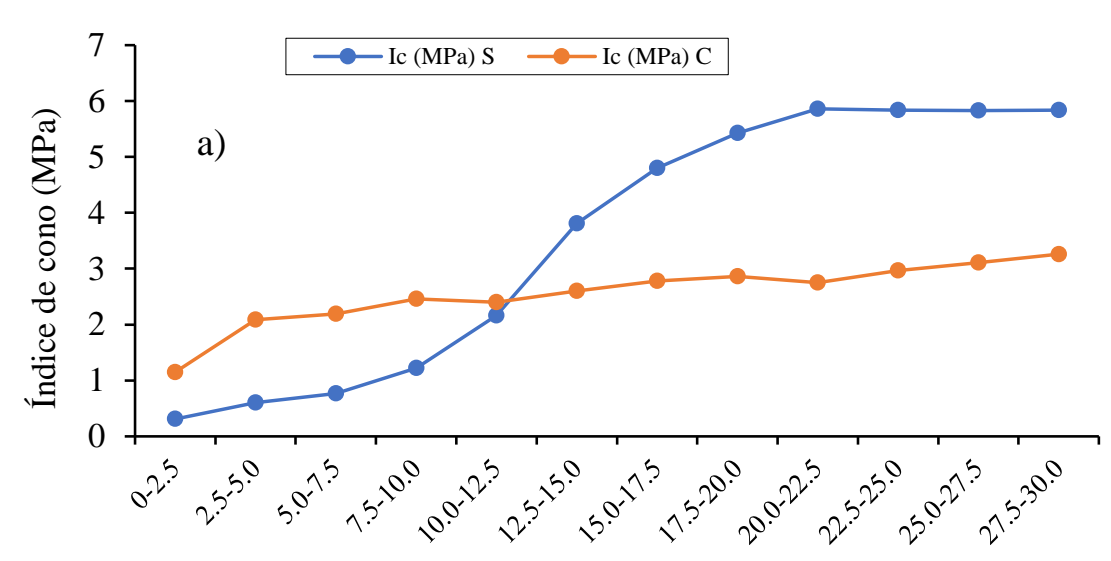

Profundidad $(\mathrm{cm})$

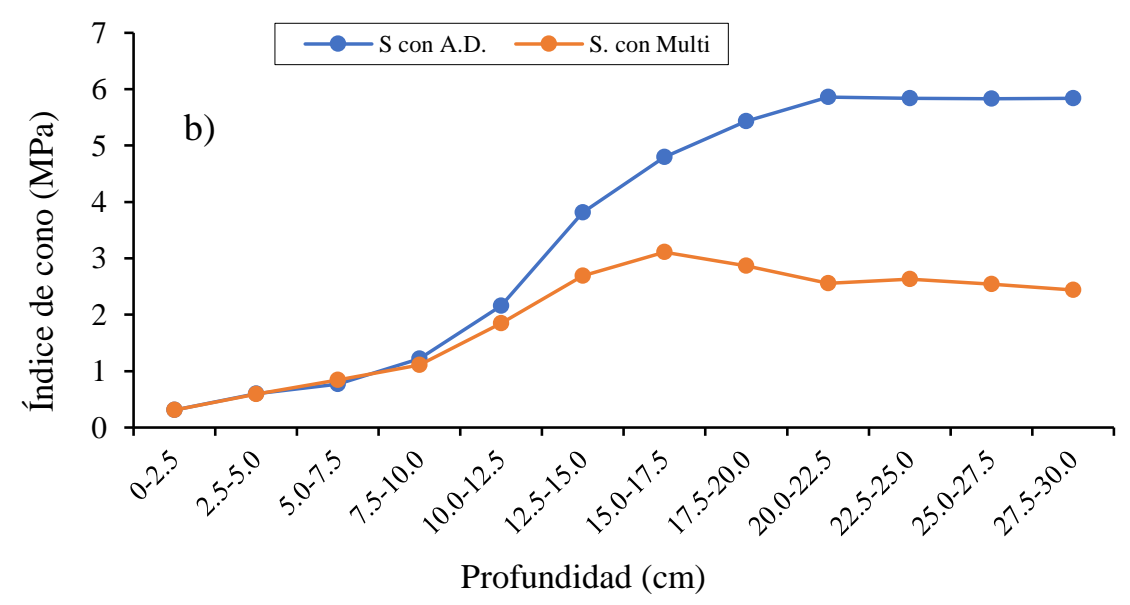

Figura 2. Índice de cono en las diferentes labranzas; a) comportamiento de la compactación del suelo después de la siembra y cosecha en labranza tradicional con arado de discos; b) comparación de índice de cono después de la siembra para labranza con arado de discos y multiarado.

En la determinación de la DA en laboratorio en siembra, las medias en las tomas de muestra cada $10 \mathrm{~cm}$ esto es, de 0-10, de 10-20 y de 20-30 cm, se obtuvieron $1.21,1.18$ y $1.21 \mathrm{Mg} \mathrm{m}^{-3}$ respectivamente, que está por debajo antes de la preparación primaria de suelo, con arado de discos. En las mediciones realizadas del Ic poscosecha siempre estuvo por encima de 1.25 MPa; a partir, 
de $5 \mathrm{~cm}$ de profundidad siempre estuvo por encima de los $2.09 \mathrm{MPa}$, llegando hasta los $3.26 \mathrm{MPa}$ en los $30 \mathrm{~cm}$ de profundidad, pero siempre por debajo del Ic registrado postsiembra, es importante plantear que la DA en los diferentes perfiles fue de 1.17 a $1.2 \mathrm{Mg} \mathrm{m}^{-3}$.

En la Figura 2b se presenta un estado comparativo del Ic postsiembra (S) en ambas preparaciones de suelo y se observa que con multiarado siempre estuvo por debajo de los $2 \mathrm{MPa}$ hasta los 22.5 (12.5) $\mathrm{cm}$, con DA de 1.17 a $1.22 \mathrm{Mg} \mathrm{m}^{-3}$, de esto se deduce que reiteradas pasadas en el suelo de cultivo es suficiente para incrementar el Ic en las capas superficiales y subsuperficiales.

En lo referente a la tecnología de labranza utilizando el multiarado AS-250, a una profundidad de trabajo de $35 \mathrm{~cm}$, el comportamiento fue un tanto diferente, ya que la situación se comportó mejor en cuanto a la cantidad de pasadas del sistema de rodaje de los tractores en las diferentes operaciones de preparación del suelo, debido a que solamente se realizaron cinco operaciones mecanizadas en el ciclo agrícola (Cuadro 3) y que al pasar el multiarado con el tractor John Deere 6603 solamente se cubrió $36.9 \%$ de la superficie que se tenía que elaborar (Figura 3). De la superficie que se tenía que elaborar se puede constatar en la Figura 2 (izquierda) la forma en la cual quedo registrada con el DGPS el tránsito del conjunto tractor-multiarado, el cual teniendo un ancho real entre ambas ruedas motrices de $1.72 \mathrm{~m}$ y con lo cual se cubrió una superficie de 6.47 ha.

Cuadro 3. Frecuencia de tráfico, en la parcela con tecnología de labranza con multiarado. Toluca, México, 2018-2019.

\begin{tabular}{ccccc}
\hline $\begin{array}{c}\text { Tipo de } \\
\text { implemento }\end{array}$ & $\begin{array}{c}\text { Ancho de } \\
\text { implemento } \\
(\mathrm{m})\end{array}$ & $\begin{array}{c}\text { Ancho de la rueda } \\
\text { trasera del tractor } \\
(\mathrm{m})\end{array}$ & $\begin{array}{c}\text { Superficie cubierta } \\
\text { por tránsito de sistema } \\
\text { de rodaje (ha) }\end{array}$ & $\begin{array}{c}\text { Frecuencia de } \\
\text { tráfico }(\%)\end{array}$ \\
\hline $\begin{array}{c}\text { Multiarado } \\
\text { Rastra (dos }\end{array}$ & 2.4 & $0.41(0.82)^{*}$ & 6.47 & 36.9 \\
pasadas) & 3 & $0.41(0.82)^{*}$ & 11.76 & 67.2 \\
Siembra & 3.2 & $0.41(0.82)^{*}$ & 4.7 & 26.9 \\
$1^{\text {ra. escarda }}$ & 2.4 & $0.47(0.94)^{*}$ & 6.84 & 39.1 \\
Total & & & 29.78 & 170.1 \\
\hline
\end{tabular}

* = ancho de ambas ruedas traseras del tractor utilizado.

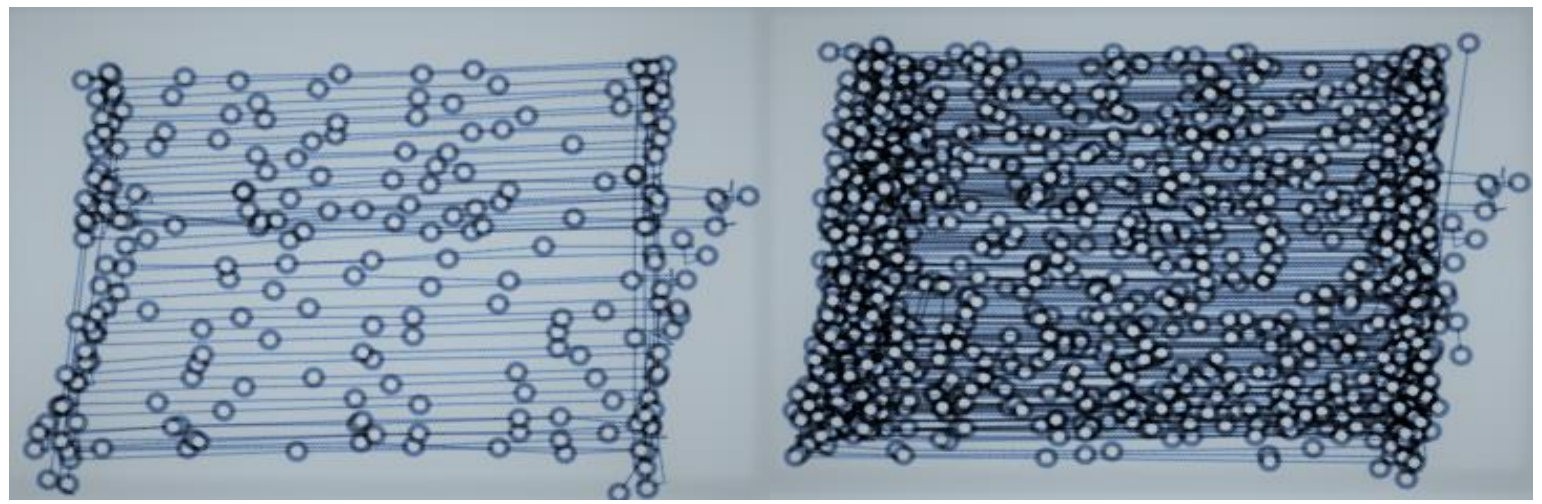

Figura. 3. Representación para la tecnología de labranza mínima. Izquierda= recorrido del tractor con multiarado; derecha = trayectorias durante el ciclo completo de cultivo del maíz. 
Después de laborar el suelo con multiarado fue necesario aplicar dos pasadas de rastra con el mismo tractor, para destruir los grades terrones que produjo este implemento y esto conllevó a que se cubriera la superficie del suelo de cultivo $67.2 \%$ y que es mucho menor que el realizado en la labranza tradicional y a partir de los $12.5 \mathrm{~cm}$ fue de $2.16 \mathrm{MPa}$. En cuanto a la densidad aparente

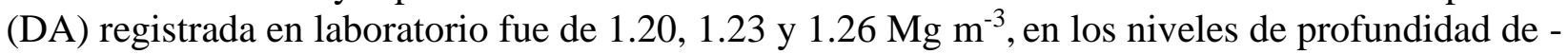
$10,10-20$ y $20-30 \mathrm{~cm}$.

En esta tecnología la pre-siembra se cubrió una superficie con el sistema de rodaje de $104.1 \%$ y al realizar un estudio comparativo con lo investigado por Kroulik et al. (2009), se puede plantear que se acerca bastante a lo realizado, aunque todas las máquinas agrícolas utilizadas por este investigador tienen anchos de labor 6 a $36 \mathrm{~m}$. Los resultados analizados con el multiarado son ostensiblemente mejores que los obtenidos en la labranza convencional.

Al utilizar una sembradora de cuatro cuerpos u órganos de trabajo $(2.4 \mathrm{~m})$, la misma estuvo acoplada a un tractor John Deere 6603 con formula 4 x 4 y el área cubierta por las huellas de las ruedas del tractor tuvo una significación de $26.9 \%$, prácticamente la misma cantidad que en la labranza tradicional; sin embargo, al compararla con las pruebas realizadas con lo realizado por Kroulik et al. 2009, el área cubierta en el experimento realizado fue 5.1\% mayor, ya que la sembradora utilizada en la República Checa es de $8 \mathrm{~m}$ de ancho, lo que da la posibilidad de tener un menor daño en la estructura al suelo y principalmente en la compactación.

La superficie cubierta por las huellas del sistema de rodaje de los tractores utilizados en esta tecnología sumó $170.1 \%$. En esta tecnología prácticamente todas las labores realizadas tienen un alto cubrimiento por las huellas de las ruedas de los tractores y en este caso Li Hong Wen et al. (2000), plantea que, a pesar de que las huellas de la rodada pueden ocupar $20 \%$ del terreno, las pérdidas en esta área pueden ser compensadas por un rendimiento más alto del cultivo.

Al comparar la Figura 1 con la Figura 3, con una frecuencia de tráfico solo del arado de disco es del orden de $91.1 \%$ con un total de $384.7 \%$, en comparación del trabajo con multiarado que es de $36.9 \%$ y en total de $170.1 \%$, donde se puede observar que en la labranza tradicional la superficie afectada por el paso de los tractores es ampliamente superada en comparación con la tecnología de labranza mínima. En base a los datos y al estudio realizado se puede plantear el tránsito rodado de maquinaria es común en la mayoría de las operaciones agrícolas, aún en sistemas de labranza cero (Tullberg, 1990).

La compactación del suelo por tránsito rodado está caracterizada por un decremento en la porosidad del suelo bajo la huella de rodamiento (Hamza y Anderson, 2005). Sin embargo, Tullberg et al. (2007) planteó que, considerando algunas combinaciones de neumáticos con los tractores utilizados, así como teniendo en cuenta las características tractivas de estos tractores, el área del campo de cultivo afectada por el tráfico del sistema de rodaje suele estar en el rango de 20 a $35 \%$.

Para el tráfico controlado, pero el caso de particular de la agricultura mexicana es que la inmensa mayoría de los implementos agrícolas utilizados son de un ancho de labor pequeño, que es uno de los grandes problemas que puede presentar el estar dañando constantemente el suelo por donde se transita, lo cual hace que se restrinja la cantidad de poros en el suelo y se registre un aumento de la compactación del suelo que pueda llegar a afectar el rendimiento de los cultivos. 
Como se puedo observar en los ensayos de campo realizados, la superficie cubierta por las huellas de los sistemas de rodaje de los tractores rebaza por mucho lo planteado por otros investigadores y a pesar de las muchas ventajas reportadas para el tránsito controlado, algunos investigadores son todavía críticos del concepto, argumentando que este sistema no ha resultado en un marcado beneficio en las propiedades del suelo o el rendimiento del cultivo (Braunack et al., 1995).

En la Figura 4a, se presenta el comportamiento del Ic durante la siembra (S con multiarado) y después de la cosecha ( $\mathrm{C}$ con multiarado) y en el perfil de $12.5 \mathrm{~cm}$ de profundidad el Ic en la siembra estuvo por encima de los $2 \mathrm{MPa}$ y contrariamente a esto en la cosecha el Ic con la excepción de $30 \mathrm{~cm}$ sobrepasó los $2 \mathrm{MPa}$ lo cual puede haberse debido a la preparación complementaria del suelo, como es la escarda. Por otro lado, González-Cueto (2009), plantea que la presión de los neumáticos muestra un incremento en la compactación a medida que aumenta la presión de inflado.
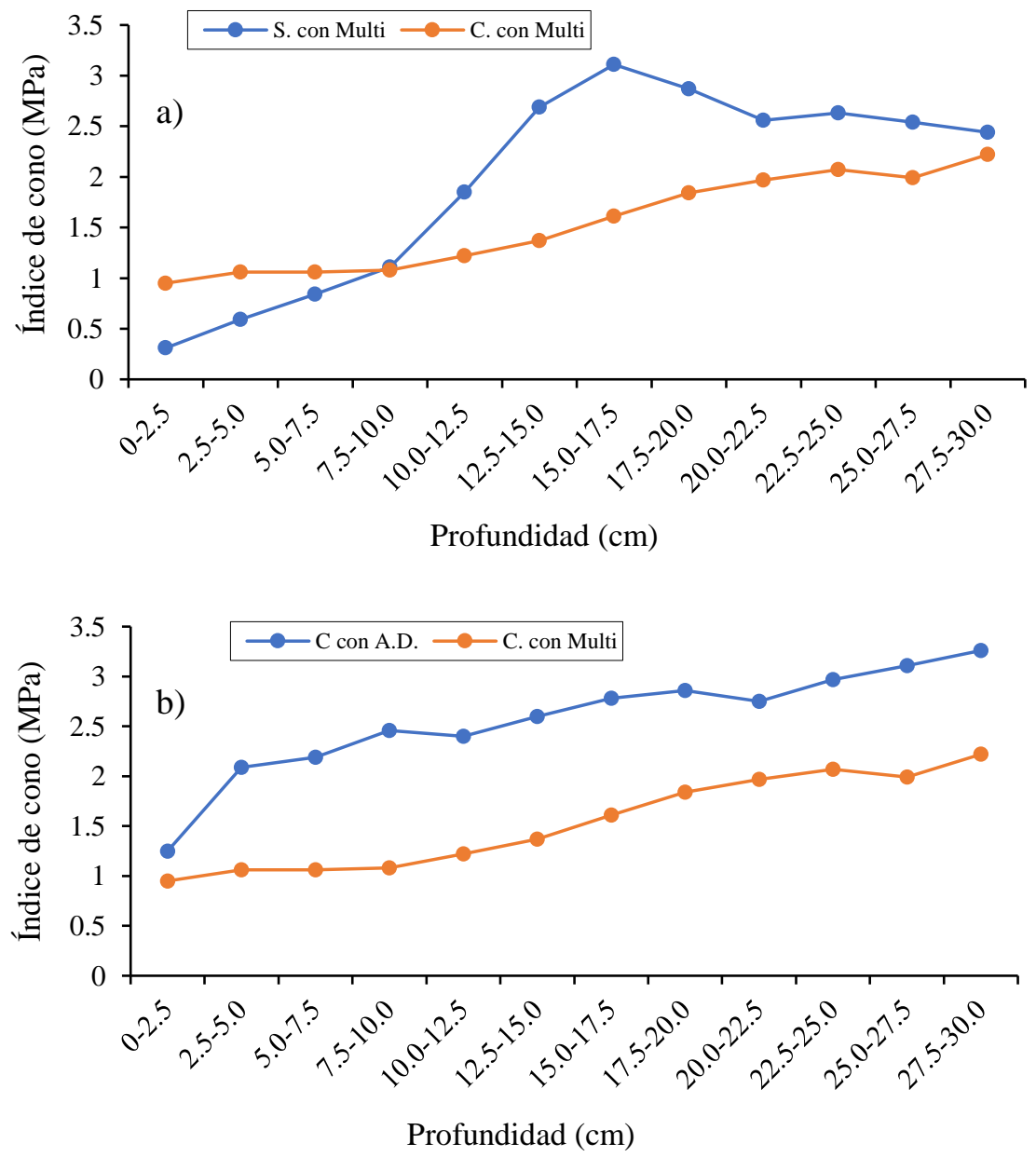

Figura 4. Índice de cono en las diferentes labranzas; a) comportamiento del índice de cono después de la siembra y cosecha en preparación del suelo con multiarado; b) comparación del índice de cono después de la cosecha entre ambas preparaciones del suelo. 
No obstante, a ello en el ensayo se mantuvo la presión en los neumáticos y el comportamiento coincide con lo planteadopor este investigador y también es el criterio de Botta et al. (2012), tanto en la capa superficial como en las profundidades de aproximadamente $30 \mathrm{~cm}$. En la Figura $4 \mathrm{~b}$, se presentan los comportamientos del Ic después de la cosecha en ambas preparaciones de suelo, donde se puede notar que los valores del Ic después de la cosecha en la preparación de suelo con multiarado es inferior a lo mostrado, donde se utilizó el arado de discos, donde este último llega a sobrepasar los $2 \mathrm{MPa}$ en prácticamente todo el perfil del suelo no siendo este el comportamiento donde se utilizó el multiarado, es importante señalar que el pase reiterado es una de las condiciones preponderantes para que exista una gran compactación, independientemente de haberse utilizado en ambos casos neumáticos radiales, en los rangos de profundidad mayores de $20 \mathrm{~cm}$ los valores del Ic tiende a crecer coincidiendo con lo encontrado por Martiren et al. (2016).

\section{Conclusiones}

En la tecnología de labranza tradicional se pasaron cuatro veces la rastra lo que sumó $205.8 \%$ de tránsito del sistema de rodaje del tractor, prácticamente dos veces la superficie que se estaba elaborando, lo cual influenció de forma negativa en la compactación del suelo; sin embargo, en la labranza con multiarado, solo se transitó $67.2 \%$. Al realizar la roturación del suelo con arado de discos se transitó $91.1 \%$ contra $36.9 \%$ del multiarado.

El tránsito del conjunto máquina tractor total en la labranza con arado de discos durante todo el ciclo agrícola fue de $384.9 \%$, prácticamente cuatro veces de la superficie cultivada y en la labranza con multiarado, fue de $170.1 \%$. En estos últimos valores mostrados se deduce que la labranza donde se utiliza el multiarado tiene ventajas, en rapidez de la preparación del suelo, menores gastos en combustible, mantenimiento del conjunto máquina tractor y daños al suelo, pues ello altera mínimamente la densidad aparente.

Los microporos del suelo y la compactación. Por lo mostrado en las mediciones del Ic, la preparación primaria con multiarado en los primeros $25 \mathrm{~cm}$ de profundidad nunca supero $3 \mathrm{MPa}$, caso contrario a la preparación de suelo con arado de discos, la cual supero los 5.5 MPa y también se observó la ventaja del multiarado después de la cosecha donde los registros del Ic fueron menores con la utilización del multiarado. La densidad aparente tuvo un comportamiento diferente al mostrado antes de comenzar la preparación de suelo, la cual fue de $1.43 \mathrm{Mg} \mathrm{m}^{-3} \mathrm{de}$ suelo o mostrando a $1.2 \mathrm{Mg} \mathrm{m}^{-3}$ terminada la siembra mecanizada en la labranza tradicional y $1.18 \mathrm{Mg} \mathrm{m}^{-3}$ después de la cosecha y en la tecnología de labranza mínima, arrojó los siguientes resultados, de $1.23 \mathrm{Mg} \mathrm{m}^{-3}$ después de la siembra y de $1.19 \mathrm{Mg} \mathrm{m}^{-3}$ después de la cosecha.

\section{Agradecimiento}

A la Universidad Autónoma del Estado de México, Estados Unidos Mexicanos, con el apoyo del proyecto 4792/2019CIB y al Consejo Nacional de Ciencias y Tecnología (CONACYT) por la beca otorgada para la realización de los estudios de Maestría, a la Facultad de Ciencias Agrícolas de la Universidad Autónoma del Estado de México; así como, al personal que labora en la maquinaria agrícola de la facultad por las facilidades y el aprecio que siempre me brindaron. 


\section{Literatura citada}

Akker, J. J. H. y Canarache, A. 2001. Two European concerted actions on subsoil compaction. Landnutzung and Landentwicklung. 42:15-22.

Botta, G. F.; Tolón-Becerra, A.; Tourn, M.; Lastra-Bravo, X. and Rivera, D. 2012. Agricultural traffic: Motion resistance and soil compaction in relation to tractor design and different soil conditions. Soil and Tillage Research. 120(2012):92-98.

Bouwman, L. A. y Arts, W. B. M. 2000. Effects of soil compaction on the relationships between nematodes, grass production and soil physical properties. Applied Soil Ecology. 14:213-222.

Braunack, M. V.; Mcphee, J. E. and Reid, D. J. 1995. Controlled traffic to increase productivity of irrigated row crops in the semi-arid tropic. Australian Journal of Experimental Agriculture. v. 35. 503-513 pp.

Cerisola, C.; Draghi, L. y Jorajuría-Collazo, D. 2015. Compactación en siembra directa. Consecuencias del tránsito durante la operación de siembra sobre la pérdida de porosidad libre al aire del suelo. Rev. De la Facultad de Agronomía, La Plata. 113(2):123-133.

Conlin, T. S. S. and Driessche R. 2000. Response of soil $\mathrm{CO}_{2}$ and $\mathrm{O}_{2}$ concentrations to forest soil compaction at the long-term soil productivity sites in central British Columbia. Canadian Journal of Soil Science. 80:625-632.

Draghi, L.; Jorajuría-Collazo, D.; Sarena, D.; Bailleres, M.; Melani, E.; Castillo, J.M.; Cerisola, C. y Palancar, T. 2015. Impacto del tránsito de dos sistemas de siembra. Montevideo Uruguay. Agrociencia. 19(2).

FAO. 2015. Los suelos están en peligro, pero la degradación puede revertirse. Roma, Italia.

González, O.; Iglesias-Coronel, C. and Herrera, M. 2009. Análisis de los factores que provocan compactación del suelo agrícola. Ciencias Técnicas Agropecuarias, Cuba. 18(2):57-63.

Hamza, M. A. and Anderson W. K. 2005. Soil compaction in cropping systems a review of the nature, causes and possible solutions. Soil \& Tillage Research. 82(2005):121-145.

Hakansson, I. and Lipiec, J. 2000. A review of the usefulness of relative bulk density values in studies of soil structure and compaction. Soil \& Tillage Research. 53(2):71-85.

Kirkegaard, J. A. (1990). Effect of compaction on the growth of pigeonpe on clay soils. Ph. D. Thesis. University of Queensland, St. Lucia, Brisbane, Australia.

Kroulik, M.; Kumhála, F.; Húls, J. and Honzik, I. 2009. The evaluation of agricultural machines field trafficking intensity for different soil tillage technologies. Soil and Tillage Research. 105:171-175.

Laureda, D. A.; Botta, G. F.; Tolón-Becerra, A. y Rosatto, H. G. 2016. Compactación del suelo inducida por la maquinaria en campos de polo en Argentina. Revista de la Facultad de Ciencias Agrarias, UNCUYO, Mendoza. 48(1):79-99.

Li, H. W.; Gao, H. W.; Chen, J. D.; Li, W. Y. and Li, R. X. 2000. Study on controlled traffic with conservative tillage. Transactions of the Chinese Society of Agricultural Engineering. 16:73-77.

Martiren, V. S.; Fonterosa, R. A.; Lastra-Bravo, X. B. and Botta, G. F. 2016. Compactación por el tráfico de la maquinaria agrícola: su efecto sobre el esfuerzo cortante del suelo y el rendimiento del cultivo de maíz (Zea mayz L.). Revista Siembra. 3:021-036.

McGarry, D.; García-Torres, L.; Benítes, J. and Martínez-Vilela, A. 2001. Tillage and soil compaction. In: First World Congress on Conservation Agriculture. (eds.). 1-5 October 2001. Natural Resource Sciences. Madrid, Spain. 281-291 pp. 
Neve, S. and Hofman G. 2000. Influence of soil compaction on carbon and nitrogen mineralization of soil organic matter and crop residues. Biology and Fertility of Soils. 30:544-549.

Richard, S. T. 2000. Development of a system for mapping the performance of agricultural field operations. End Thesis, unpublished Cranfield University at Silsoe, Bedford, UK.

Soil Science Society of America. 2013. Glossary of Soil Science Terms. Madison, WI, USA. 92 p.

Tullberg, J. 1990. Why control field traffic? In: Proceedings of Queensland Department of primary industries Soil Compaction Workshop, Toowoomba Australia. 41-62 pp.

Tullberg, J. N.; Yule, D. Y. and Garry, D. Mc. 2007. Controlled traffic farming-from research to adoption in Australia. Soil and Tillage Research. 97(2):272-281.

Tullberg, J. 2010. Tillage, traffic and sustainability a challenge for ISTRO Soil and Tillage Research. 111:26-32.

Wild, A. 1992. Condiciones del suelo y desarrollo de las plantas según Russell. Ediciones MundiPrensa. 1025 p. 